\title{
Polar lattice vibrations and phase transition dynamics in $\mathrm{Pb}\left(\mathrm{Zr}_{1-x} \mathrm{Ti}_{x}\right) \mathrm{O}_{3}$
}

\author{
E. Buixaderas, ${ }^{1, *}$ D. Nuzhnyy, ${ }^{1}$ J. Petzelt, ${ }^{1}$ Li Jin(靳立), ${ }^{2}$ and D. Damjanovic ${ }^{2}$ \\ ${ }^{1}$ Department of Dielectrics, Institute of Physics, Academy of Sciences of the Czech Republic, 18221 Prague 8, Czech Republic \\ ${ }^{2}$ Ceramics Laboratory, Swiss Federal Institute of Technology_EPFL, Lausanne, Switzerland
}

(Received 8 September 2011; revised manuscript received 8 October 2011; published 15 November 2011)

\begin{abstract}
Infrared (IR) reflectivity spectra of nominally pure $\mathrm{Pb}\left(\mathrm{Zr}_{1-x} \mathrm{Ti}_{x}\right) \mathrm{O}_{3}$ ceramics with different $\mathrm{Ti} / \mathrm{Zr}$ concentration ( $x=0.42-0.58$ ) were measured and evaluated, along with the time-domain terahertz transmittance spectra in the temperature range $10 \mathrm{~K}-900 \mathrm{~K}$. The temperature dependence of the low-frequency vibrations, related to $\mathrm{Pb}$ atoms, was analyzed in terms of two overdamped modes-a soft mode and an anharmonic hopping central mode-in the cubic and high-temperature ferroelectric phase and three main vibrations in the low-temperature ferroelectric phase with the doubled unit cell: two E-symmetry modes (the soft mode and a mode corresponding to antiphase vibrations of neighboring $\mathrm{Pb}$ atoms in the terahertz range) and the antiferrodistortive mode producing the antiphase tilts of the oxygen octahedra. The last bare mode is not IR active, but it becomes activated by coupling with the soft mode. As predicted by theory, the intrinsic permittivity of $\mathrm{Pb}\left(\mathrm{Zr}_{1-x} \mathrm{Ti}_{x}\right) \mathrm{O}_{3}$ has a maximum at the morphotropic phase boundary, although this represents just a small percentage of the total permittivity at lower frequencies. Its maximum is linked to the softening of the anharmonic vibrations of $\mathrm{Pb}$ ions, perpendicular to the polarization, and shifts from $x=0.48$ at room temperature to $x=0.52$ at $10 \mathrm{~K}$.
\end{abstract}

DOI: $10.1103 /$ PhysRevB.84.184302

PACS number(s): 78.30.-j, 63.20.-e, 77.80.bg

\section{INTRODUCTION}

Lead zirconate titanate ceramics $\mathrm{Pb}\left(\mathrm{Zr}_{1-x} \mathrm{Ti}_{x}\right) \mathrm{O}_{3}$ (PZT 100 $-X / X$, where $X=100 x$ ) have been well known since their exceptional optical and dielectric properties were revealed and reviewed in the seventies by Land et al. ${ }^{1}$ However, their real structure and the relationship between the local structure and macroscopic properties are far from being understood.

The Ti-rich part of the PZT phase diagram shows tetragonal symmetry, space group $P 4 m m(x>0.48)$. The Zr-rich part is rhombohedral (space group $R 3 c$ ) for $\mathrm{Zr}$ contents between $\sim 50 \%$ and $90 \%{ }^{1}$ Samples with still higher content in $\mathrm{Zr}$ show more complex structure due to the presence of an orthorhombic antiferroelectric state, which is characteristic for the parent compound $\mathrm{PbZrO}_{3} .{ }^{2}$ Ceramics with Ti content near $48 \%$ show a maximum in the piezoelectric response at room temperature, which is the reason of the wide use of these ceramics in electronics.

The discovery of a monoclinic bridging phase between the tetragonal and the rhombohedral sides of the phase diagram near the morphotropic phase boundary (MPB) in PZT $52 / 48$ was thought to be the cause of the high piezoelectric coefficients due to a polarization rotation mechanism. ${ }^{3,4}$ In this phase, the polarization vector is free to rotate to preserve the monoclinic symmetry within a plane defined by its (001) tetragonal and (111) rhombohedral direction. How much it will rotate depends on the transverse permittivity.

The monoclinic phase has been subject of a great controversy. In the past, due to the impossibility of growing large, single-domain PZT crystals at the MPB, most experiments were done on ceramics. Therefore, past $\mathrm{x}$-ray diffraction, neutron elastic scattering, and Raman experiments on ceramic samples did not present reliable proofs of a true zero-field monoclinicity due to the high anharmonic thermal parameters in their structural refinements, coherence effects, and the presence of nanodomains. ${ }^{5,6}$ It was also pointed out that the excellent piezoelectric properties of lead-based materials with MPBs are due to adaptive phases composed of fine nanotwinned domains of different symmetries (rhombohedral and tetragonal), ${ }^{7}$ which look monoclinic just in the average. Then the piezoelectric effect would be not intrinsic; rather, it would be caused by domain switching at the nanoscale level and favored by the applied electric field. This switching was found experimentally in PZT and could explain its high piezoelectric values. ${ }^{6}$ However, domains coexistence did not ruled out the presence of a monoclinic phase mixed with other phases. ${ }^{8,9}$ The recent development of bigger single PZT crystals has allowed researchers to perform new structural studies in which coexistence of at least two phases seems to be the answer to the PZT structure. ${ }^{10,11}$

An important factor in PZT is the dynamic, structural, and chemical disorder in MPB samples, which causes the local and macroscopic symmetries to differ. $\mathrm{Pb}$ displacements were found in rhombohedral and tetragonal PZT samples, ${ }^{5}$ creating a monoclinic distortion in the unit cell. ${ }^{12} \mathrm{~A}$ macroscopic monoclinic phase could then be explained by condensation of the $\mathrm{Pb}$ shifts in a particular direction in the structure. ${ }^{13}$ However, open questions remain regarding the symmetry of PZT: It is still unclear whether the monoclinic phase is a real macroscopic phase or just a monoclinic distortion of the parent tetragonal and rhombohedral phases to relieve strain, and we still do not know whether the local chemical or structural disorder leads to the monoclinicity when it is correlated at a larger scale.

To answer some of these questions and find the local symmetry of PZT ceramics, Raman spectra of PZT ceramics were repeatedly studied. ${ }^{14-19}$ The monoclinic phase was supported by Souza-Filho et al.; ${ }^{17}$ however, in their studies, no considerations about the cation disorder or doubling of the unit cell at low temperatures were made. Recently, some of us have suggested that extra modes present in tetragonal PZT ceramics can be due to the double occupancy of the B site, in the general $\mathrm{ABO}_{3}$ perovskite lattice, by $\mathrm{Zr}$ and $\mathrm{Ti}$ atoms, to dynamic disorder of $\mathrm{Pb}$, or to doubling of the unit cell by oxygen octahedra tilting. ${ }^{20}$ All of these effects activate new 
phonon modes in the Raman and infrared (IR) spectra. ${ }^{16,20,21}$ PZT ceramics with spatially graded composition around the MPB were also studied in situ by micro-Raman mapping, ${ }^{22}$ and it was found that the relative intensity of the two E(TO2) components, due to the double occupancy of the B site, is related to the amount of $\mathrm{Ti}$ in the lattice and therefore presents a measure of the tetragonality of PZT.

IR studies on PZT are scarcer. The first far IR reflectivity study on PZT was performed for the rhombohedral PZT $75 / 25$ sample by Železný et al., ${ }^{23}$ and room temperature reflectivity dependence on the composition was studied by Sivasubramanian et $a l .{ }^{24}$ Transmission studies were done on thin films by Fedorov et al. ${ }^{25}$ and on ceramics by Araújo and colleagues, ${ }^{26-28}$ but only above $400 \mathrm{~cm}^{-1}$. Our group published several papers on IR properties of lanthanum-doped PZT (PLZT) ${ }^{20,29,30}$ and doped soft and hard PZT, ${ }^{21}$ but to understand the behavior of polar phonons, systematic IR studies on undoped PZT are more suitable. This paper aims to improve understanding of the polar phonons in PZT. Here, we present IR reflectivity and time-domain terahertz $(\mathrm{THz})$ transmission data from $20 \mathrm{~K}$ to $900 \mathrm{~K}$ on a set of undoped PZT ceramics with compositions crossing the MPB with $x=0.42$ to 0.58 . Symmetry analysis of the IR active modes is presented, as well as the temperature dependences of their parameters.

\section{EXPERIMENTAL DETAILS}

All PZT ceramics were prepared by a conventional solidstate process using a standard mixed-oxide route and $\mathrm{PbO}$, $\mathrm{TiO}_{2}$, and $\mathrm{ZrO}_{2}$ starting powders of $99.9 \%$ chemical purity. PZT ceramics with $x=0.42$ and 0.58 were prepared at the École Polytechnique Fédérale in Lausanne. The density of the samples was higher than $97 \%$ of the theoretical one, and the grain size was $\sim 10 \mu \mathrm{m}$. Samples showed $\mathrm{x}$-ray diffraction patterns consistent with tetragonal and rhombohedral symmetries. More experimental details can be found in Ref. 31. PZT ceramics with $0.45 \leqslant x \leqslant 0.52$ were prepared at the Materials Research Lab of Pennsylvania State University. A more detailed description, including diffraction studies, can be found in Ref. 4. Our dielectric and calorimetric measurements of the samples showed a decrease of the temperature of the ferroelectric (FE) phase transition, $T_{C}$, from $696 \mathrm{~K}$ to $650 \mathrm{~K}$ on decreasing the Ti content from 0.58 to 0.42 , in agreement with Jaffe et al. ${ }^{32}$ The purity of the starting powders from both sources was good enough not to introduce impurities that could significantly affect the dielectric response above $100 \mathrm{GHz}$, as shown in Ref. 21.

IR reflectivity measurements were performed on polished ceramic discs ( $7 \mathrm{~mm}$ in diameter and $1 \mathrm{~mm}$ thick) using a Fourier-transform IR spectrometer, the Bruker IFS 113v. Room and high-temperature spectra were taken in the range $30-3000 \mathrm{~cm}^{-1}$ with a resolution of $2 \mathrm{~cm}^{-1}$ and a deuterated triglycine sulfate pyroelectric detector. For high temperatures (300 K-900 K), a commercial oven Specac P/N 5850 was used. For low-temperature measurements $(10 \mathrm{~K}-300 \mathrm{~K})$, we used an Optistat continuous flow (CF) cryostat (Oxford Instruments) with the sample mounted in a He gas bath. The polyethylene windows of the cryostat reduced the accessible frequency range to $30-650 \mathrm{~cm}^{-1}$ at low temperatures. Detection was done by a He-cooled (1.5 K) Si bolometer.

Time-domain THz spectroscopy (TDTS) transmission measurements were carried out on thin, polished, plane-parallel samples in a similar temperature range. Samples with $x=0.45$, 0.48 , and 0.52 had thicknesses $d=47$ and $98 \mu \mathrm{m}$ for high and low temperatures, respectively; sample $x=0.42$ had $d=58 \mu \mathrm{m}$; and sample $x=0.58$ had $d=73 \mu \mathrm{m}$.
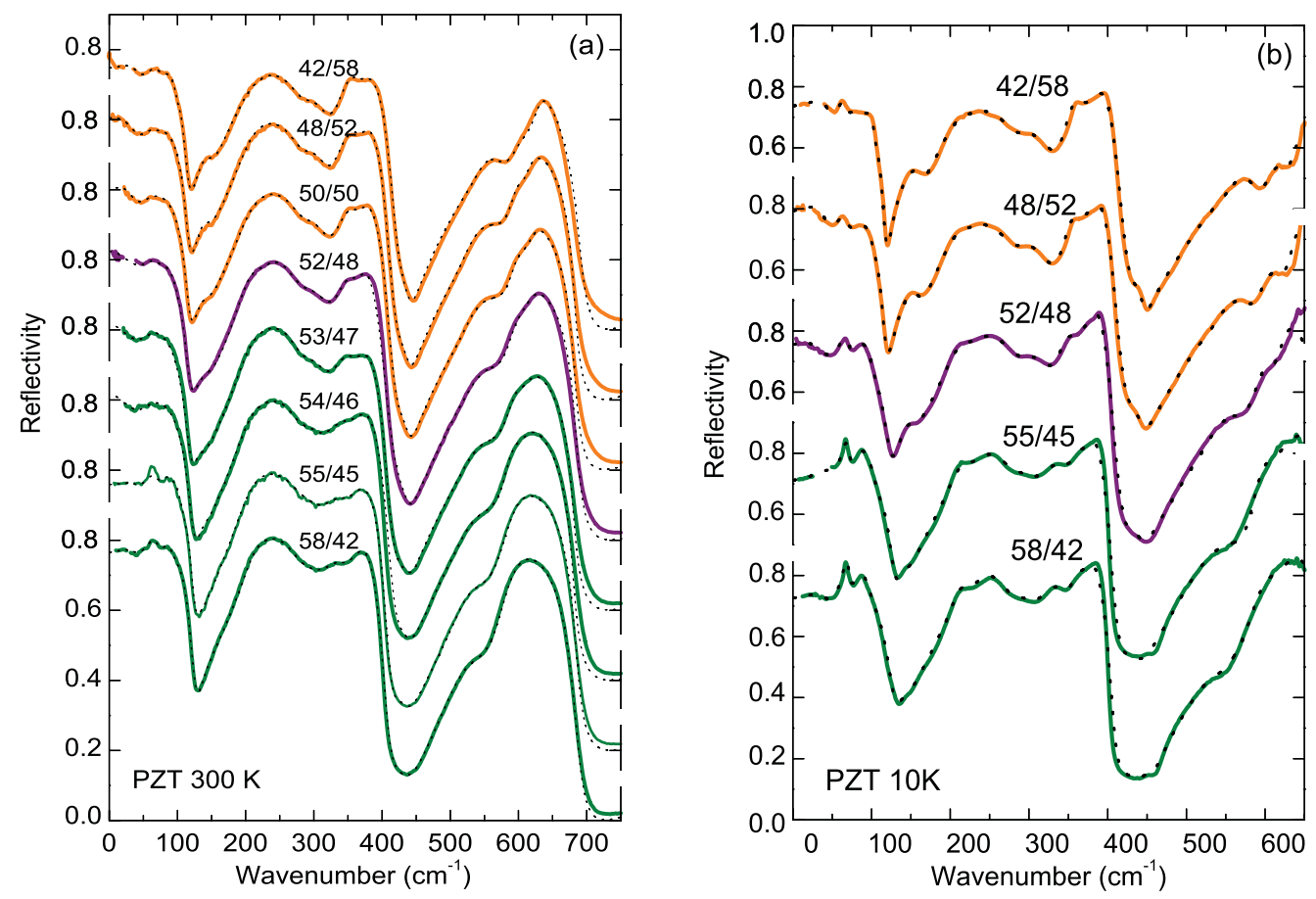

FIG. 1. (Color online) IR reflectivity spectra of several PZT ceramics at (a) $300 \mathrm{~K}$ and (b) $10 \mathrm{~K}$, together with their fits using Eqs. (1) and (2). Experimental data below $30 \mathrm{~cm}^{-1}$ correspond to the calculated reflectivity from the TDTS experiment. 
A custom-made $\mathrm{THz}$ transmission spectrometer was used to obtain the complex dielectric response from $\sim 5$ to $20 \mathrm{~cm}^{-1}$ with a resolution of $0.5 \mathrm{~cm}^{-1}$. At higher frequencies, the samples were opaque. An Optistat CF cryostat with Mylar windows was used for measurements down to $20 \mathrm{~K}$. An adapted commercial high-temperature cell Specac P/N 5850 was used to heat the samples up to $900 \mathrm{~K}$.

\section{EXPERIMENTAL RESULTS}

The reflectivity spectra of PZT ceramics obtained from the IR experiment were fitted using the factorized oscillator model of the dielectric function ${ }^{33}$

$$
\begin{gathered}
\varepsilon^{*}(\omega)=\varepsilon^{\prime}(\omega)-\mathrm{i} \varepsilon^{\prime \prime}(\omega)=\varepsilon_{\infty} \prod_{j=1}^{n} \frac{\omega_{\mathrm{L} j}^{2}-\omega^{2}+\mathrm{i} \omega \gamma_{\mathrm{L} j}}{\omega_{\mathrm{T} j}^{2}-\omega^{2}+\mathrm{i} \omega \gamma_{\mathrm{T} j}}, \\
\Delta \varepsilon_{j}=\frac{\varepsilon_{\infty}}{\omega_{\mathrm{T} j}^{2}} \frac{\prod_{k}\left(\omega_{\mathrm{L} k}^{2}-\omega_{\mathrm{T} j}^{2}\right)}{\prod_{k \neq j}\left(\omega_{\mathrm{T} k}^{2}-\omega_{\mathrm{T} j}^{2}\right)},
\end{gathered}
$$

where $\varepsilon_{\infty}$ is the permittivity at frequencies much higher than all polar phonon frequencies; $\omega_{\mathrm{T} j}$ and $\omega_{\mathrm{L} j}$ are the transverse and longitudinal frequencies, respectively, of the $j$ th phonon mode; and $\gamma_{\mathrm{T} j}$ and $\gamma_{\mathrm{L} j}$ are their respective damping constants. $\Delta \varepsilon_{j}$ refers to their contributions to the static permittivity. The complex dielectric permittivity $\varepsilon^{*}(\omega)=\varepsilon^{\prime}(\omega)-\mathrm{i} \varepsilon^{\prime \prime}(\omega)$ is related to the reflectivity spectrum $R(\omega)$ by

$$
R(\omega)=\left|\frac{\sqrt{\varepsilon^{*}(\omega)}-1}{\sqrt{\varepsilon^{*}(\omega)}+1}\right|^{2} .
$$

Our fitting procedure enables us to fit simultaneously the reflectivity from the IR range and the complex dielectric spectra $\left(\varepsilon^{\prime}\right.$ and $\left.\varepsilon^{\prime \prime}\right)$ from the TDTS experiment, obtaining in this way rather reliable parameters even in the very far IR range.

The reflectivity spectra of PZT ceramics, together with the corresponding fits using Eqs. (1) and (2), are shown in Fig. 1 at room temperature and at $10 \mathrm{~K}$. The reflectivity spectra below $30 \mathrm{~cm}^{-1}$ were calculated from the TDTS data. Inspection of the figure shows a rather monotonous evolution of the three main IR bands on changing the Ti content. These bands are associated to the three main bands of the cubic $\mathrm{ABO}_{3}$ perovskites labeled Last, Slater, and Axe modes, whose eigenvectors are mainly related to the A-, B-, and O-atom

\begin{tabular}{|c|c|c|c|c|c|c|c|c|c|c|}
\hline \multirow[b]{2}{*}{ Band } & \multirow[b]{2}{*}{ Label } & \multicolumn{3}{|c|}{ PZT 48/52 } & \multicolumn{3}{|c|}{ PZT 52/48 } & \multicolumn{3}{|c|}{ PZT 55/45 } \\
\hline & & $\omega$ & $\gamma$ & $\Delta \varepsilon$ & $\omega$ & $\gamma$ & $\Delta \varepsilon$ & $\omega$ & $\gamma$ & $\Delta \varepsilon$ \\
\hline \multirow{8}{*}{ Last } & $\mathrm{E}\left(\mathrm{TO}^{\mathrm{b}}\right)$ & 29.1 & 46.0 & 220 & 30.1 & 66.0 & 360 & 36.3 & 49.2 & 140 \\
\hline & $\mathrm{E}\left(\mathrm{LO}^{\mathrm{c}}\right)$ & 45.4 & 45.7 & & 56.4 & 73.4 & & 51.7 & 52.4 & \\
\hline & $\mathrm{E}(\mathrm{TO} 1)$ & 64.0 & 56.5 & 53.0 & 69.5 & 50.5 & 19.0 & 79.2 & 36.7 & 24.0 \\
\hline & $\mathrm{E}(\mathrm{LO} 1)$ & 117.2 & 20.5 & & 117.9 & 22.4 & & 123.6 & 19.0 & \\
\hline & $\mathrm{AFD}(\mathrm{TO})$ & 69 & 20.1 & 14.0 & 67.2 & 20.5 & 17.2 & 64.2 & 20.2 & 23.5 \\
\hline & $\mathrm{AFD}(\mathrm{LO})$ & 65 & 19.9 & & 68.3 & 23.6 & & 71.2 & 18.2 & \\
\hline & $\mathrm{A}_{1}(\mathrm{TO} 1)$ & 135.0 & 40.8 & 2.7 & 134.6 & 45.6 & 3.0 & 134.2 & 36.6 & 0.8 \\
\hline & $\mathrm{A}_{1}(\mathrm{LO} 1)$ & 144.9 & 52.9 & & 146.5 & 49.6 & & 138.8 & 40.0 & \\
\hline \multirow[t]{12}{*}{ Slater } & $\mathrm{E}(\mathrm{TO} 2)$ & 202.2 & 47.6 & 18.6 & 203.3 & 42.0 & 16.0 & 202.8 & 44.0 & 16.5 \\
\hline & $\mathrm{E}(\mathrm{LO} 2)$ & 415.8 & 30.1 & & 413.0 & 41.9 & & 410.3 & 24.2 & \\
\hline & $\mathrm{E}\left(\mathrm{TO} 2^{\prime}\right)$ & 228.0 & 12.0 & 0.2 & 228.0 & 29.4 & 0.5 & 230.0 & 21.5 & 0.5 \\
\hline & $\mathrm{E}\left(\mathrm{LO} 2^{\prime}\right)$ & 227.8 & 11.9 & & 226.7 & 29.5 & & 228.9 & 22.6 & \\
\hline & $\mathrm{E}\left(\mathrm{TO}-\mathrm{F}_{2 u}\right)$ & 291.1 & 48.9 & 0.6 & 285.0 & 52.7 & 0.5 & 280.0 & 31.4 & 0.3 \\
\hline & $\mathrm{E}\left(\mathrm{LO}-\mathrm{F}_{2 u}\right)$ & 285.4 & 52.9 & & 280.2 & 50.1 & & 279.5 & 30.8 & \\
\hline & $\mathrm{GR}^{\mathrm{d}}$ & 322.5 & 55.9 & & 331.5 & 58.3 & & 308.8 & 89.6 & \\
\hline & $\mathrm{A}_{1}(\mathrm{TO} 2)$ & 335.2 & 48.3 & 0.8 & 337.5 & 43.4 & 0.3 & 322.0 & 70.3 & 0.7 \\
\hline & $\mathrm{A}_{1}(\mathrm{LO} 2)$ & 438.7 & 22.3 & & 436.8 & 23.8 & & 433.2 & 33.5 & \\
\hline & $\mathrm{A}_{1}\left(\mathrm{TO} 2^{\prime}\right)$ & 371.9 & 31.4 & 0.06 & 370.0 & 37.8 & 0.05 & 353.1 & 51.7 & 0.05 \\
\hline & $\mathrm{A}_{1}\left(\mathrm{LO}^{\prime}\right)$ & 370.3 & 33.2 & & 368.1 & 45.0 & & 347.3 & 49.8 & \\
\hline & $\mathrm{GR}^{\mathrm{d}}$ & 436.2 & 32.4 & & 435.9 & 33.9 & & 426.7 & 34.7 & \\
\hline \multirow[t]{6}{*}{ Axe } & $\mathrm{E}(\mathrm{TO} 3)$ & 530.0 & 75.7 & 1.9 & 518.0 & 78.4 & 1.6 & 512.8 & 75.2 & 1.5 \\
\hline & $\mathrm{E}(\mathrm{LO} 3)$ & 682.3 & 13.3 & & 685.2 & 26.0 & & 685.0 & 16.3 & \\
\hline & $\mathrm{GR}^{\mathrm{d}}$ & 593.7 & 58.7 & & 581.1 & 72.6 & & 567.0 & 80.7 & \\
\hline & $\mathrm{A}_{1}(\mathrm{TO} 3)$ & 597.0 & 35.3 & 0.07 & 586.3 & 51.2 & 0.1 & 577.0 & 49.8 & 0.2 \\
\hline & $\mathrm{A}_{1}(\mathrm{LO} 3)$ & 708.0 & 73.0 & & 703.0 & 62.2 & & 696.0 & 54.8 & \\
\hline & $\mathrm{GR}^{\mathrm{d}}$ & 687.0 & 38.2 & & 685.0 & 51.3 & & 686.8 & 39.3 & \\
\hline
\end{tabular}
displacements, respectively. ${ }^{34}$

TABLE I. Parameters of the IR modes fitted at $300 \mathrm{~K}$ for three PZT compositions. ${ }^{\mathrm{a}}$

\footnotetext{
${ }^{\mathrm{a}} \omega$ and $\gamma$ are given in reciprocal centimeters.

${ }^{\mathrm{b}} \mathrm{TO}$ stands for transverse optic modes.

${ }^{\mathrm{c}} \mathrm{LO}$ stands for longitudinal optic modes.

${ }^{\mathrm{d}} \mathrm{GR}$ stands for geometric resonances appearing in ceramics.
} 


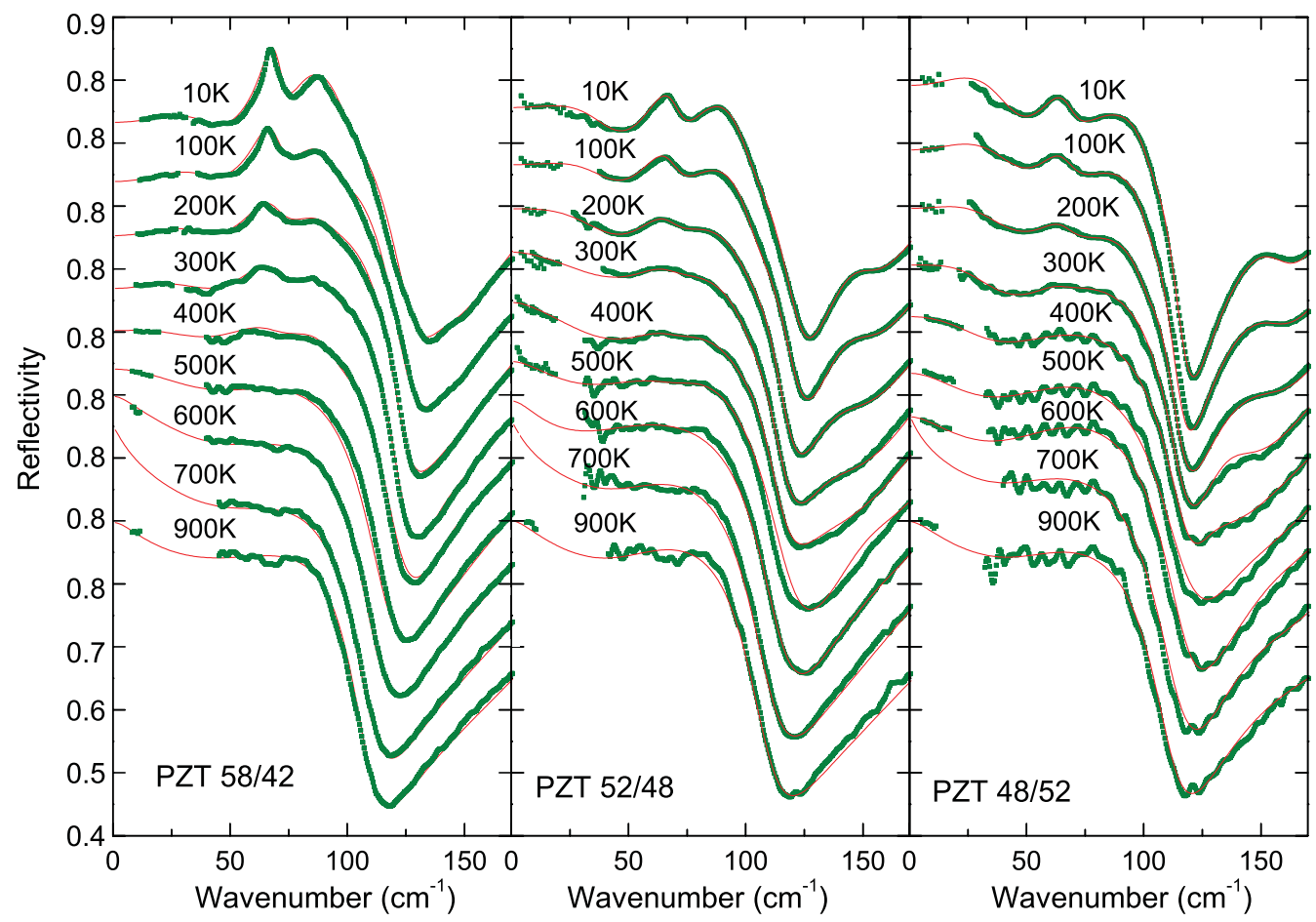

FIG. 2. (Color online) Low-frequency IR reflectivity spectra of PZT with $x=0.42,0.48$, and 0.52 at different temperatures, together with their fits using Eqs. (1) and (2). Data below $30 \mathrm{~cm}^{-1}$ correspond to the calculated reflectivity from the TDTS experiment.

At room temperature, the first (lowest frequency) band, related to $\mathrm{Pb}$ vibrations, is quite broad. However, in samples with higher $\mathrm{Zr}$ content (on the rhombohedral side of the phase diagram), a peak above $50 \mathrm{~cm}^{-1}$ develops. At low temperatures [Fig. 1(b)], this peak is better seen. The second band is prevailingly related to the $\mathrm{Zr} / \mathrm{Ti}$ vibrations against the oxygen octahedra (Slater mode). Its shape also reveals changes according to the $\mathrm{Zr}$ / Ti ratio: samples with higher Ti content (tetragonal) develop a peak $\sim 150 \mathrm{~cm}^{-1}$ and a deeper minimum above $300 \mathrm{~cm}^{-1}$. These two effects are more noticeable at low temperatures; in addition, more substructure appears in all studied compositions. The highest-frequency (Axe mode) band, related to bending of the oxygen octahedra, also shows a change of its shape with Ti content. Table I shows the parameters of the IR fitted modes for three selected compositions: in the tetragonal side, at the MPB, and in the rhombohedral side of the phase diagram. Labeling of the modes was done according to the results of the effective medium approximation fit in tetragonal $\mathrm{PLZT}^{20}$ and Raman results on a single $\mathrm{PbTiO}_{3}$ crystal. ${ }^{35}$

Figure 2 shows the temperature dependences of the reflectivity spectra of three PZT samples $(x=0.42,0.48$, and $0.52)$ below $200 \mathrm{~cm}^{-1}$. In this frequency range, the first band at high temperatures, in the cubic phase, corresponds to the Last mode. ${ }^{34}$ On cooling, this band splits into the $\mathrm{E}+\mathrm{A}_{1}$ doublet corresponding to the $\mathrm{FE}$ phase $\left(\mathrm{E}\right.$ and $\mathrm{A}_{1}$ refer to the symmetries of the irreducible representations, see Table II). The E-symmetry component remains always below $100 \mathrm{~cm}^{-1}$, and the $\mathrm{A}_{1}$-symmetry component appears above $120 \mathrm{~cm}^{-1}$. The $A_{1}$ component is better seen in tetragonal samples, and on cooling, it hardens up to $140 \mathrm{~cm}^{-1}$. The flat or increasing level of the reflectivity below $40 \mathrm{~cm}^{-1}$ toward lower frequencies, calculated from the TDTS experiment, indicates the presence of a central mode (CM) component. ${ }^{36}$ On further cooling, the IR spectra of all samples reveal a new mode $\left(\omega=60-70 \mathrm{~cm}^{-1}\right)$. This mode appears, in the rhombohedral samples, near the temperature where the doubling of the unit cell due to the oxygen octahedra tilt occurs. Tetragonal samples as PZT 48/52 and 42/58 were not supposed to undergo such a tilt, although indications of the prolongation of the tilting of the oxygen octahedra toward the tetragonal side were found for samples with Ti content up to $x=0.53 \cdot{ }^{19,37}$ The new mode is also present in these samples upon cooling, although it appears at lower temperatures [see Figs. 1(b) and 2].

Permittivity and loss spectra obtained from the combined fit of the $\mathrm{THz}$ complex dielectric data and the IR reflectivity using Eqs. (1) and (2) are depicted in Figs. 3 and 4 for several PZT ceramics at different temperatures. Fig. 3 presents the experimental dielectric permittivity divided in two parts. Left panels show the $\mathrm{THz}$ range, together with the calculated permittivities up to $100 \mathrm{~cm}^{-1}$. Higher frequencies are shown in right panels in a different scale. Dielectric spectra in Figs. 3 and 4 show an excitation in the $\mathrm{THz}$ range, which dramatically changes with temperature. For all PZT samples, the maximum value of the extrapolated permittivity corresponds to temperatures near or above $T_{C}$, and then, on cooling, the permittivity decreases. A similar effect can be seen in the intensity of the loss peak between 10 and $30 \mathrm{~cm}^{-1}$. The temperature behavior of its frequency is alike for all PZT samples: the maximum of the loss peak shifts to higher frequencies below $T_{C}$. Notwithstanding the qualitatively similar behavior in all samples, there is an important difference in the dielectric contribution of the $\mathrm{THz}$ excitation $\left(\Delta \varepsilon_{\mathrm{THz}}\right)$ when the $\mathrm{Zr} / \mathrm{Ti}$ ratio changes: around room temperature, its highest value corresponds to compositions near the MPB, and then $\Delta \varepsilon_{\mathrm{THz}}$ decreases when going away 


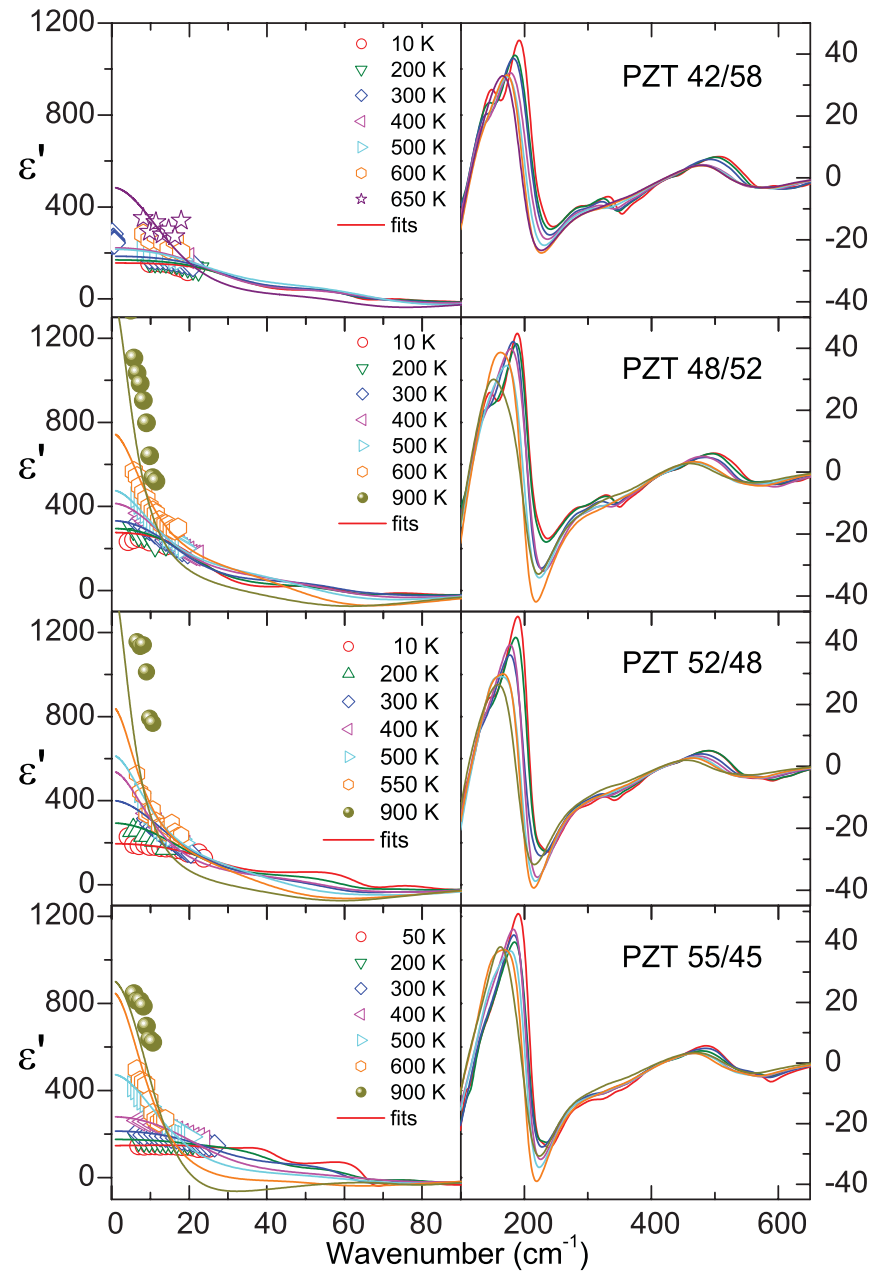

FIG. 3. (Color online) Permittivity of several PZT ceramics at different temperatures obtained from the fit of the reflectivity. Experimental points show the TDTS experiment, and lines show fits using Eqs. (1) and (2).

from the MPB. The probable origin of this excitation is discussed next.

\section{DISCUSSION}

The classic factor-group analysis for PZT with rhombohedral symmetry predicts $3 \mathrm{~A}_{1}(\mathrm{IR}, \mathrm{R})+4 \mathrm{E}(\mathrm{IR}, \mathrm{R})+1 \mathrm{~A}_{2}(-)$ optic modes in the high-temperature FE phase (space group $R 3 \mathrm{~m}$ ), and $4 \mathrm{~A}_{1}(\mathrm{IR}, \mathrm{R})+9 \mathrm{E}(\mathrm{IR}, \mathrm{R})+5 \mathrm{~A}_{2}(-)$ optic modes in the low-temperature phase (space group $R 3 c$, doubled primitive unit cell). ${ }^{14,16}$ The factor-group analysis predicts $3 \mathrm{~A}_{1}(\mathrm{IR}, \mathrm{R})+$ $4 \mathrm{E}(\mathrm{IR}, \mathrm{R})+1 \mathrm{~B}_{1}(\mathrm{R})$ modes in the tetragonal FE $P 4 m m$ phase $^{14}$ and $4 \mathrm{~A}_{1}(\mathrm{IR}, \mathrm{R})+8 \mathrm{E}(\mathrm{IR}, \mathrm{R})+1 \mathrm{~B}_{1}(\mathrm{R})+2 \mathrm{~B}_{2}(\mathrm{R})+5 \mathrm{~A}_{2}(-)$ in the doubled-cell tetragonal $I 4 \mathrm{~cm}$ phase (Table II). Mode labels refer to the symmetries of the irreducible representations and their activities-IR, Raman (R) or inactive (-) - are shown in brackets. This analysis considers ordered structures with one type of B-site atoms in the perovskite lattice. However, PZT ceramics have two different atoms in the B position. The distribution of $\mathrm{Zr} / \mathrm{Ti}$ atoms in PZT might not be homogeneous (by Monte Carlo simulations, $\mathrm{Zr} / \mathrm{Ti}$-rich clusters of $\sim 10-20$ unit cells could be present); ${ }^{38}$ therefore, they do not need to behave like one effective atom. Due to the different masses,

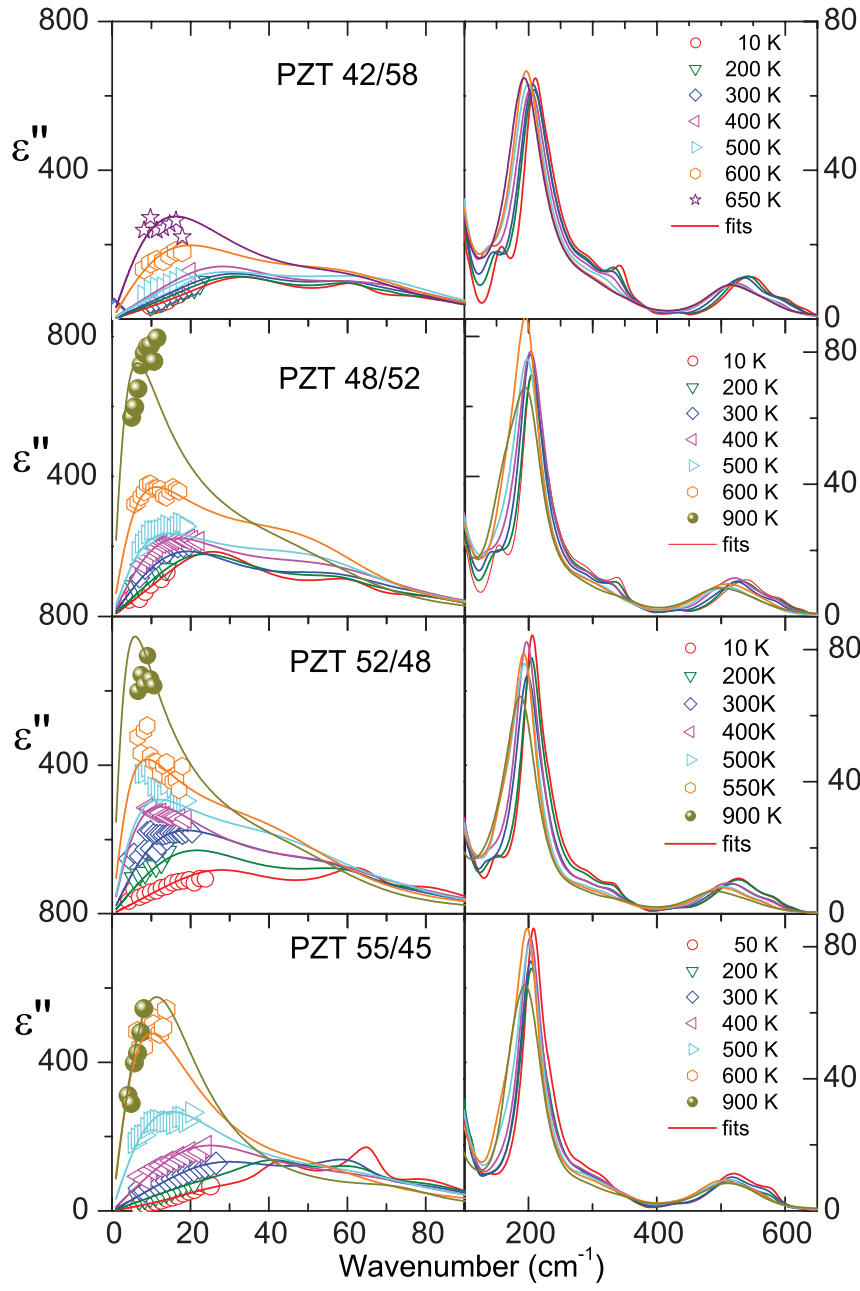

FIG. 4. (Color online) Loss spectra of several PZT ceramics at different temperatures obtained from the fit of the reflectivity. Experimental points show the TDTS experiment, and lines show fits using Eqs. (1) and (2).

separate vibrations corresponding to both $\mathrm{Zr}-\mathrm{O}_{6}$ and $\mathrm{Ti}-\mathrm{O}_{6}$ octahedra can be found (similar to the two-mode behavior in mixed crystals). ${ }^{39}$ Therefore, modes arising from the Slater and Axe modes, where the atoms in the B position are involved, can be doubled. This effect was already found in tetragonal PZT and PLZT compositions, ${ }^{20,21}$ and it explains why the total number of modes in the tetragonal FE phases of PZT is higher than expected, without the need of decreasing the macroscopic symmetry.

We took this two-atom occupancy into consideration to calculate the number of modes expected in the real PZT. Results of the symmetry analysis are presented in Table II, including the expected number of modes in the monoclinic groups $\mathrm{Cm}$ and $\mathrm{Cc}$. The FE groups of PZT above room temperature, $R 3 m, C m$, and $P 4 m m$, give 9, 13, and 9 IR active modes, respectively. The cubic $\mathrm{F}_{1 u}$ Slater band (vibrations from B-site atoms) splits into $2 \mathrm{~A}_{1}+2 \mathrm{E}$ modes for both the tetragonal and rhombohedral sides due to the two-atom occupancy. The monoclinic $\mathrm{Cm}$ phase with one effective $\mathrm{Ti} / \mathrm{Zr}$ atom would have just $2 \mathrm{~A}^{\prime}+1 \mathrm{~A}^{\prime \prime}$ modes. We have used five modes to fit the Slater band (see Table I), 2E(TO2) + $2 \mathrm{~A}_{1}(\mathrm{TO} 2)$ coming from the $\mathrm{Zr}$ and $\mathrm{Ti}$ atoms and another $\mathrm{E}$ 


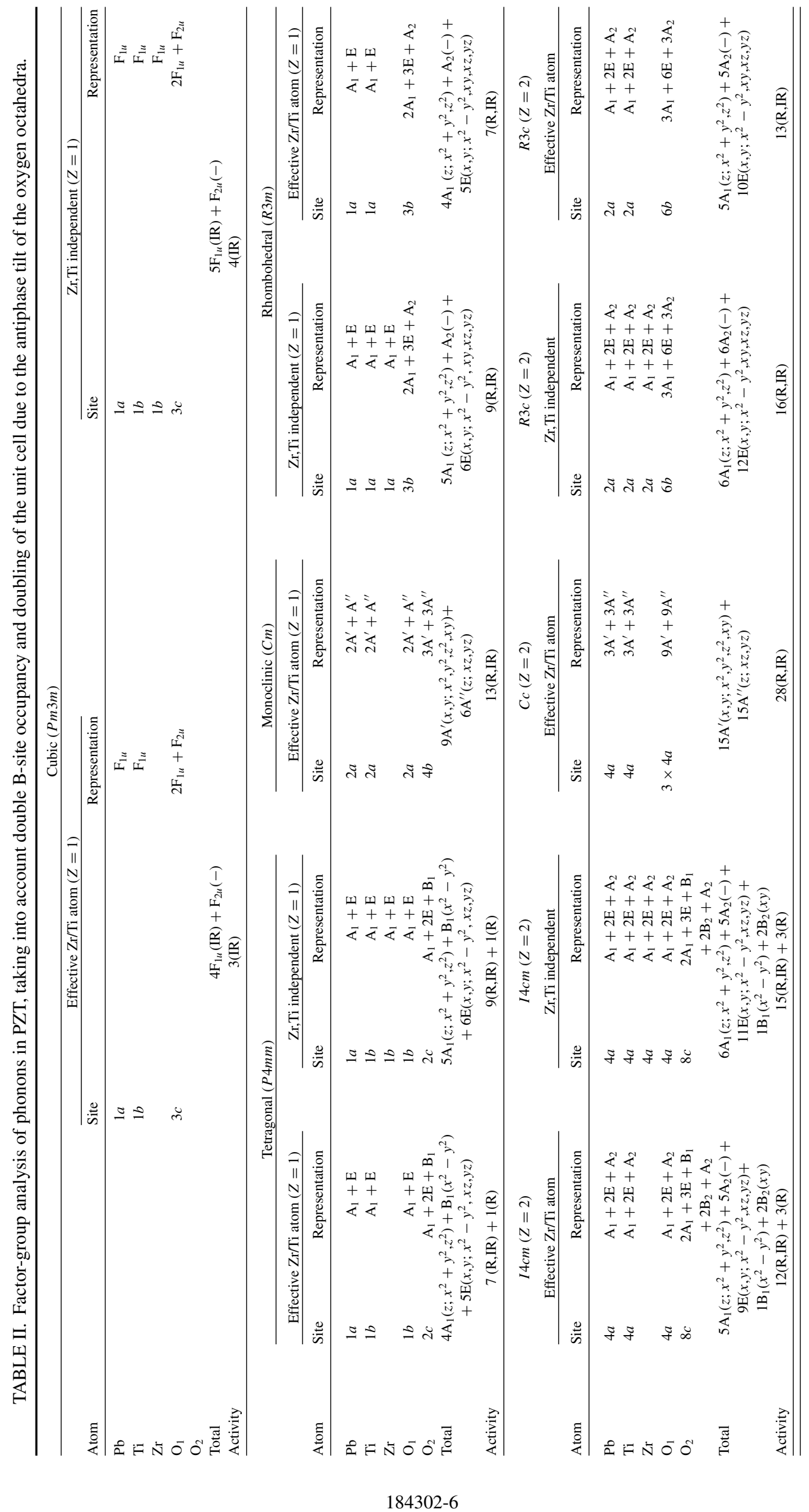




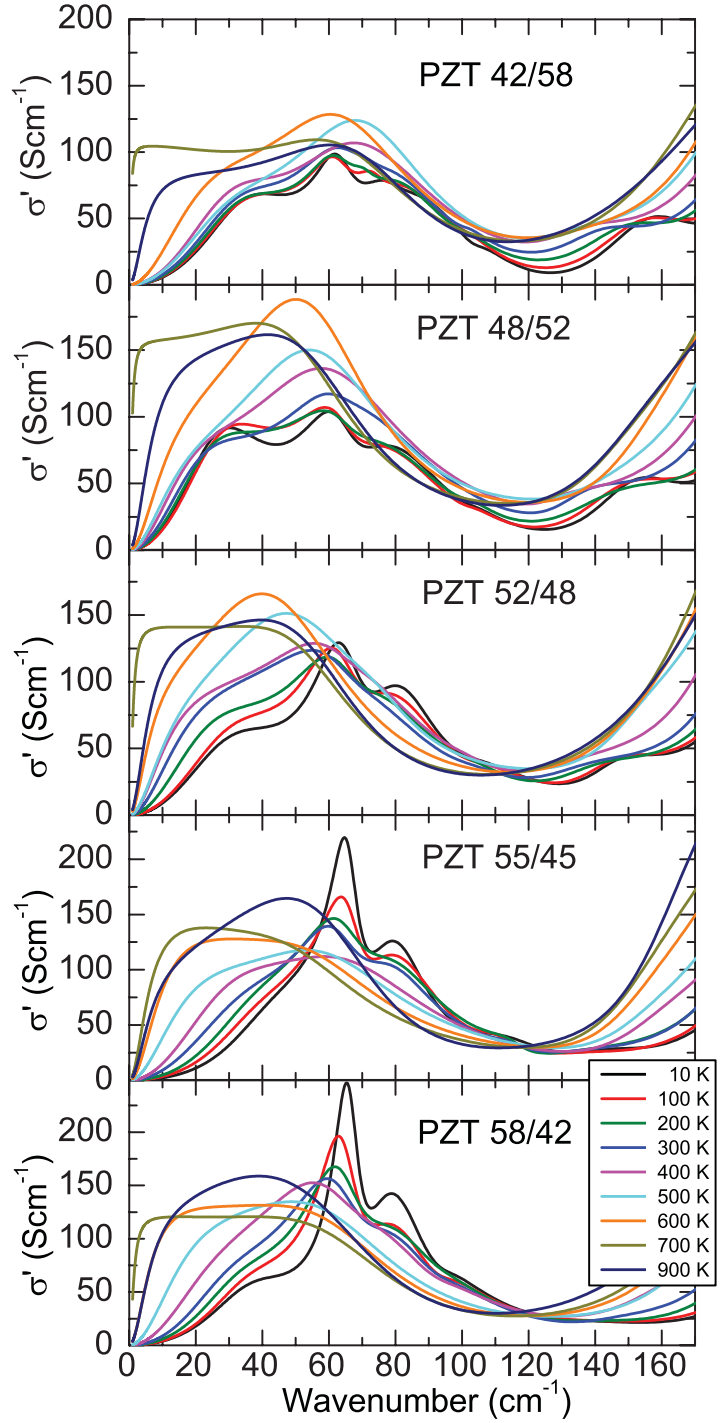

FIG. 5. (Color online) Temperature dependences of the optical conductivity spectra of PZT ceramics, calculated from the fits using Eqs. (1) and (2).

mode stemming from the silent $\mathrm{F}_{2 u}$ cubic mode. So, the $R 3 m$ and $P 4 \mathrm{~mm}$ symmetries satisfactorily fit all samples, even the MPB sample. In total, we have used nine IR modes plus two low-frequency extra modes, present in all compositions. The IR probe does not distinguish between $R 3 \mathrm{~m}$ and $P 4 \mathrm{~mm}$ groups because they present the same number of IR active modes, but it could be able to reveal the $\mathrm{Cm}$ group, which was not confirmed even at low temperatures.

The fit was performed in a systematic way for all samples to find possible differences due to the $\mathrm{Zr} / \mathrm{Ti}$ substitution, and special emphasis was put on revealing new modes at low temperatures. For this purpose, all samples were considered equivalent in the cubic phase: three main bands were fit plus an extra excitation in the $\mathrm{THz}$ range to reproduce the measured $\mathrm{THz}$ values. The accuracy of this fitting procedure is, however, limited at high temperatures: below $100 \mathrm{~cm}^{-1}$, we used an overdamped oscillator that fits $\varepsilon^{\prime}$ and $\varepsilon^{\prime \prime}$ measured in the $\mathrm{THz}$ range and an oscillator (phonon of $\mathrm{F}_{1 u}$ symmetry) capable to fit the IR reflectivity. A fit with just one oscillator in this

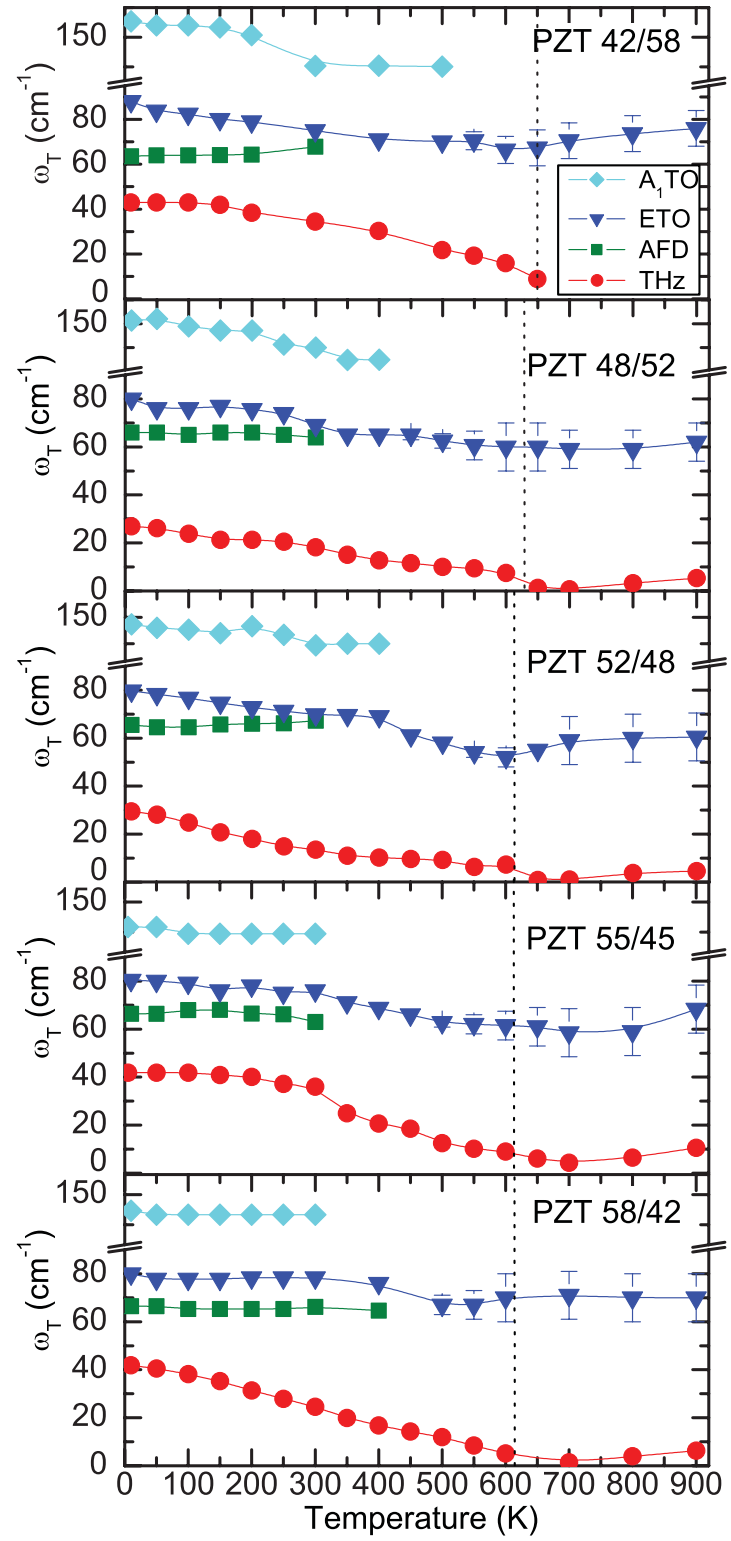

FIG. 6. (Color online) Temperature dependence of the transverse frequencies of the $\mathrm{Pb}$-related modes, calculated from the fits using Eqs. (1) and (2).

range failed to provide good results. The drawback of our fit is the large error bars of the $\mathrm{F}_{1 u}$ (TO1) frequency above $T_{C}$ and therefore of its dielectric strength $\Delta \varepsilon$ (see Figs. 6 and 7). To overcome this, we constrained some parameters: the longitudinal frequency of the overdamped mode was kept below $60 \mathrm{~cm}^{-1}$ to fit a shallow minimum in the reflectivity, and the damping constant $\gamma_{\mathrm{T}}$ at high temperatures was kept between 50 and $60 \mathrm{~cm}^{-1}$, which otherwise would tend to behave anomalously. Below $T_{C}$, due to the decrease of the damping constants the accuracy of the fit appreciably increases and error bars are within the symbols. Results of the fit are presented in Figs. 5-9.

Figure 5 depicts the temperature dependence of the real part of the optical conductivity $\sigma^{\prime}(\omega)=\left(2 \pi \mathrm{c} \varepsilon_{0}\right) \omega \varepsilon^{\prime \prime}$ (where $c$ is the speed of light in vacuum, $\varepsilon_{0}$ is the permittivity of vacuum, and the frequency $\omega$ is given in reciprocal centimeters), 


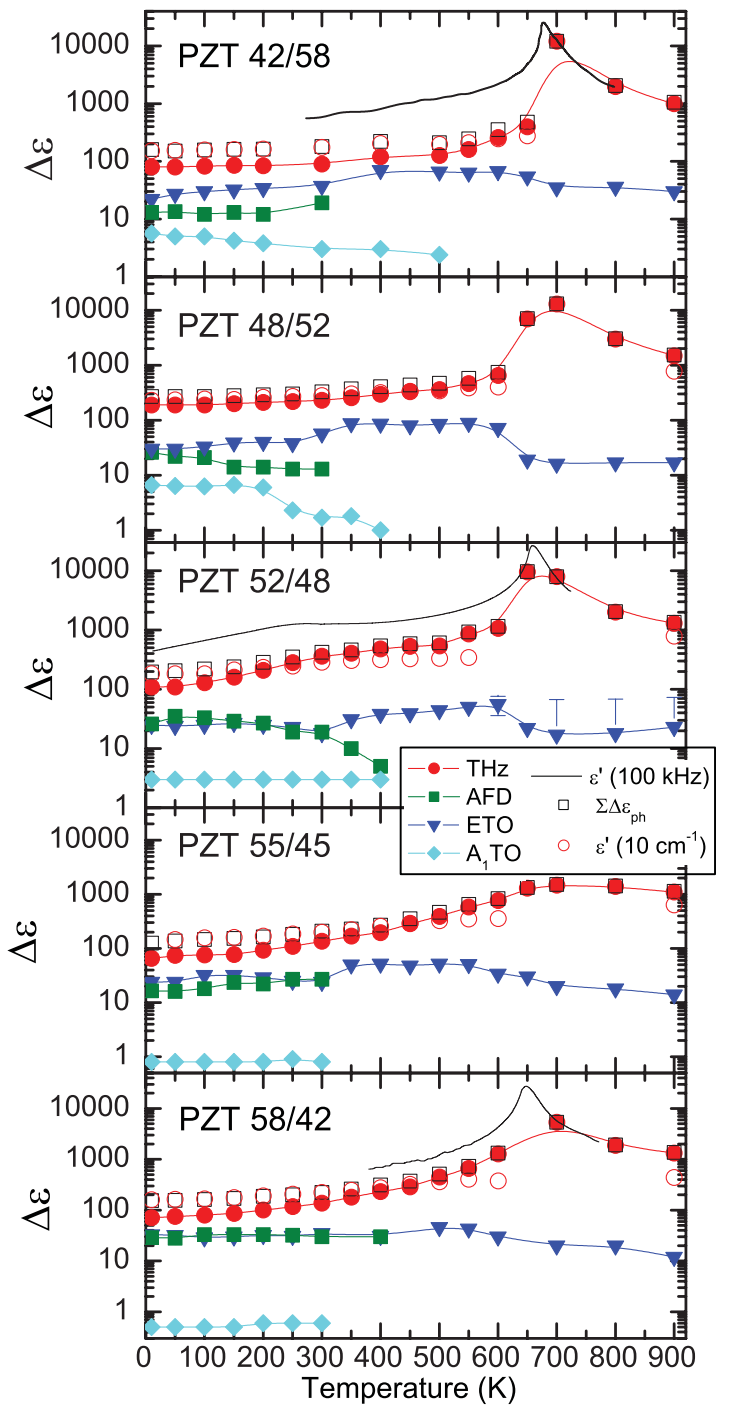

FIG. 7. (Color online) Temperature dependence of the permittivity and dielectric contribution of the low-frequency modes.

below $100 \mathrm{~cm}^{-1}$ for several PZT compositions. This range corresponds to modes split out of the cubic Last band. The temperature dependence of the conductivity reveals that all PZT compositions show similar behavior on cooling. In the paraelectric phase, a broad excitation is present composed of two contributions: the $\mathrm{F}_{1 u}$ mode near $60 \mathrm{~cm}^{-1}$ and a CM component below $20 \mathrm{~cm}^{-1}$. On cooling toward $T_{C}$, the $\mathrm{CM}$ softens to very low frequencies, producing broad optical conductivity spectra near $T_{C}$. This softening also induces the huge increase of the permittivity toward $T_{C}$. Then below $T_{C}$, in the FE phase, two maxima appear: one corresponds to the $\mathrm{E}(\mathrm{TO} 1)$ mode and the other one to an excitation in the $\mathrm{THz}$ range. On further cooling, another peak develops in the $60-70 \mathrm{~cm}^{-1}$ range (the temperature when this peak appears is called $T_{\text {tilt }}$ ). At the lowest temperature, three maxima are clearly seen for all samples: the low-frequency mode stemmed from the $\mathrm{THz}$ component, which hardens from 10 to $30-40 \mathrm{~cm}^{-1}$; the sharper mode appearing $\sim 65 \mathrm{~cm}^{-1}$; and the $\mathrm{E}$ (TO1) mode, which shows a moderate hardening up to $80 \mathrm{~cm}^{-1}$ on cooling from $T_{\text {tilt }}$. These modes are labeled THz, antiferrodistortive (AFD), and ETO, respectively.
The temperature dependences of the transverse frequencies $\omega_{\mathrm{T}}$ corresponding to the modes below $100 \mathrm{~cm}^{-1}$ are shown in Fig. 6. The ETO mode has a frequency ranging from 60 to $80 \mathrm{~cm}^{-1}$, with a weaker temperature dependence. The mode in the $\mathrm{THz}$ range account for the high values of the permittivity (seen as a huge peak in $\varepsilon^{\prime \prime}$ in Fig. 3). On cooling, this mode hardens from 10 to $30-40 \mathrm{~cm}^{-1}$, depending on the composition. Finally, the AFD mode $\left(\omega \approx 65 \mathrm{~cm}^{-1}\right)$ appears on cooling in the temperature range $300 \mathrm{~K}-400 \mathrm{~K}$ in all samples.

The damping constants of these modes are quite high: above room temperature, they have values $\sim 60 \mathrm{~cm}^{-1}$, but even at lowest temperatures they range from 20 to $40 \mathrm{~cm}^{-1}$ (values at room temperature are in Table I). The only exception is the AFD mode, appearing as a sharper peak in the rhombohedral samples [see Fig. 1(b)]. The MPB 52/48 sample and the tetragonal $48 / 52$ one have particularly large values of the damping constants, which indicate a higher anharmonicity or disorder of the MPB structure and its surroundings. This fact causes the special character of these modes, overdamped in most of the temperature range. These high damping constants are a typical feature of PZT ceramics, which causes additional difficulty in interpreting the results. According to the last diffraction results on PZT crystals, ${ }^{8-11}$ coexistence of monoclinic domains with tetragonal and/or rhombohedral domains is probably the answer of the PZT structure. Our results do not confirm a macroscopic monoclinic symmetry; however, due to the high values of the damping constants, even at $10 \mathrm{~K}$, a hidden monoclinicity could be present in the MPB samples in a very small fraction of the volume, not enough to be detected as the splitting of the E-symmetry modes.

The dielectric contributions of the modes related to $\mathrm{Pb}$ vibrations (Last band) are shown in Fig. 7. The most striking result is the dielectric contribution of the $\mathrm{THz}$ mode, which is the highest in the whole temperature range. This mode carries the main dielectric anomaly at the FE phase transition. In contrast, the ETO mode shows much a smaller anomaly.

The oscillator strengths of the modes stemming from the Last band are presented in Fig. 8. All samples show a value near $500000 \mathrm{~cm}^{-2}$ remaining reasonably constant on cooling. When taking a look into the oscillator strengths of the individual oscillators, we can see that these are not constant. The oscillator strengths of the THz mode $\left(f_{\mathrm{THz}}=\omega_{\mathrm{THz}}{ }^{2} \Delta \varepsilon_{\mathrm{THz}}\right)$ decrease on cooling: this means that this excitation is coupled with another (probably the ETO mode) and a transfer of strengths occurs between them. Tetragonal samples have a lower $f_{\mathrm{THz}}$ value in the FE phase. In rhombohedral samples, this change takes place at lower temperatures near $T_{\text {tilt }}$. In the respective tilted phases, the three E-symmetry modes show similar oscillator strengths, and their eigenvectors are probably hybridized. The total oscillator strength of the Last band is higher for tetragonal samples. This fact is partially explained by the $\mathrm{A}_{1}$ (TO1) strength, which is very weak in the rhombohedral samples but increases gradually with the $\mathrm{Ti}$ content.

The mode in the $60-80 \mathrm{~cm}^{-1}$ range is assigned to the soft $\mathrm{E}$ (TO1) mode, which correspond to vibrations of $\mathrm{Pb}$ atom against the octahedra network perpendicular to the polarization. This mode is expected to be the soft mode (SM) of the FE transition in tetragonal/rhombohedral perovskites; 


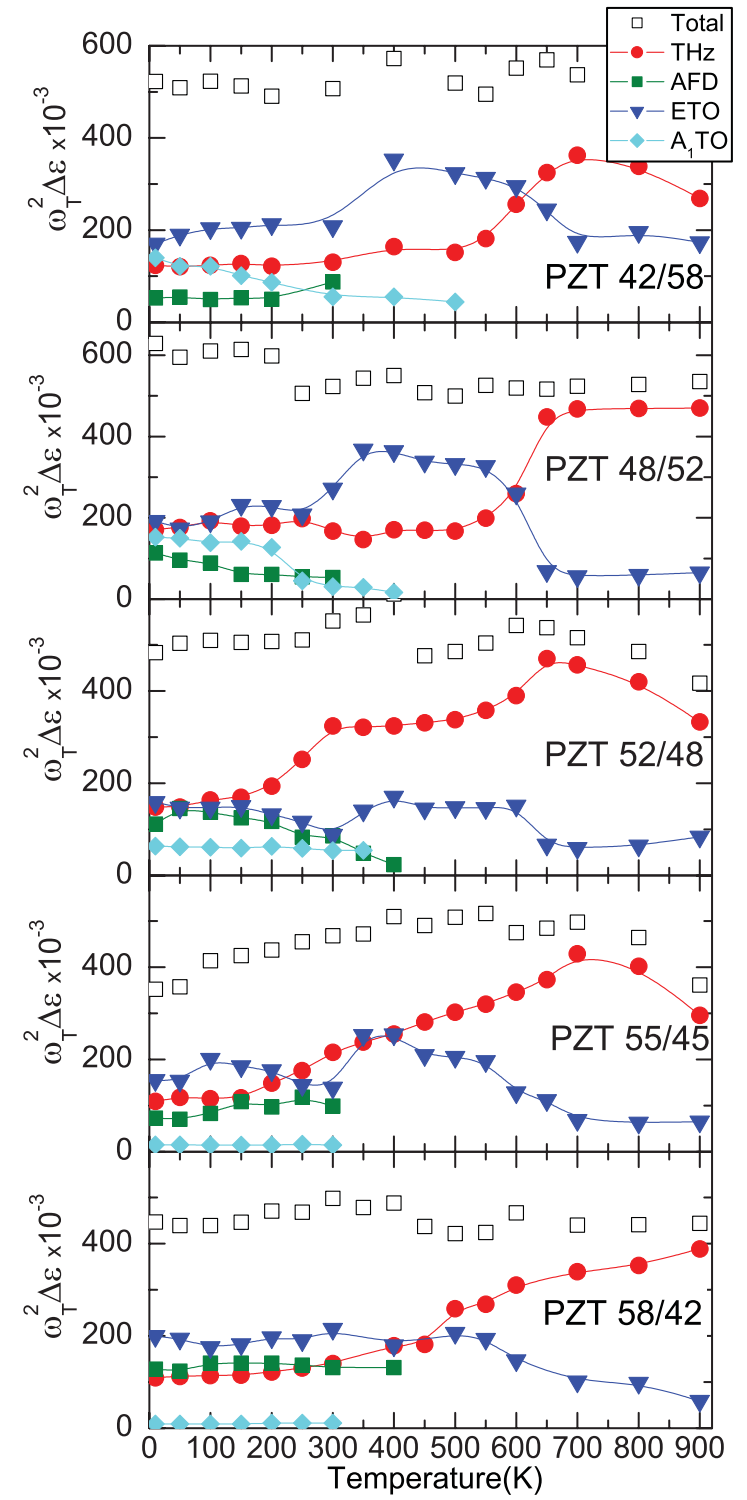

FIG. 8. (Color online) Temperature dependence of the oscillator strengths of the low-frequency modes.

however, in PZT, it softens only slightly. We believe that this is due to its coupling to the other two low-frequency modes.

The new mode appearing on cooling in the rhombohedral PZT ceramics (AFD mode) is related to the AFD $R_{25}$ mode (tilt of the oxygen octahedra about the polarization axis). This mode stems from the Brillouin zone corner where it is nonpolar; however, in the doubled unit cell, it can be activated by the $\mathrm{Pb}-\mathrm{O}$ interaction, as shown by dynamical simulations. ${ }^{40}$ This AFD transition is accompanied by a small dielectric peak in the permittivity in high Zr-content PZT. ${ }^{41}$ As the AFD mode was also found in the tetragonal PZT samples, we suggest that PZT with $0.52 \leqslant x \leqslant 0.58$ also undergoes a transition to a tilted phase at low temperatures. This phase was also predicted by first-principles calculations, and the assigned space group was $I 4 \mathrm{~cm} .{ }^{42}$ Therefore, it seems that in the PZT compositional phase diagram, the line of the oxygen tilting transition crosses continuously the MPB toward the tetragonal side. A suggested phase diagram is plotted in

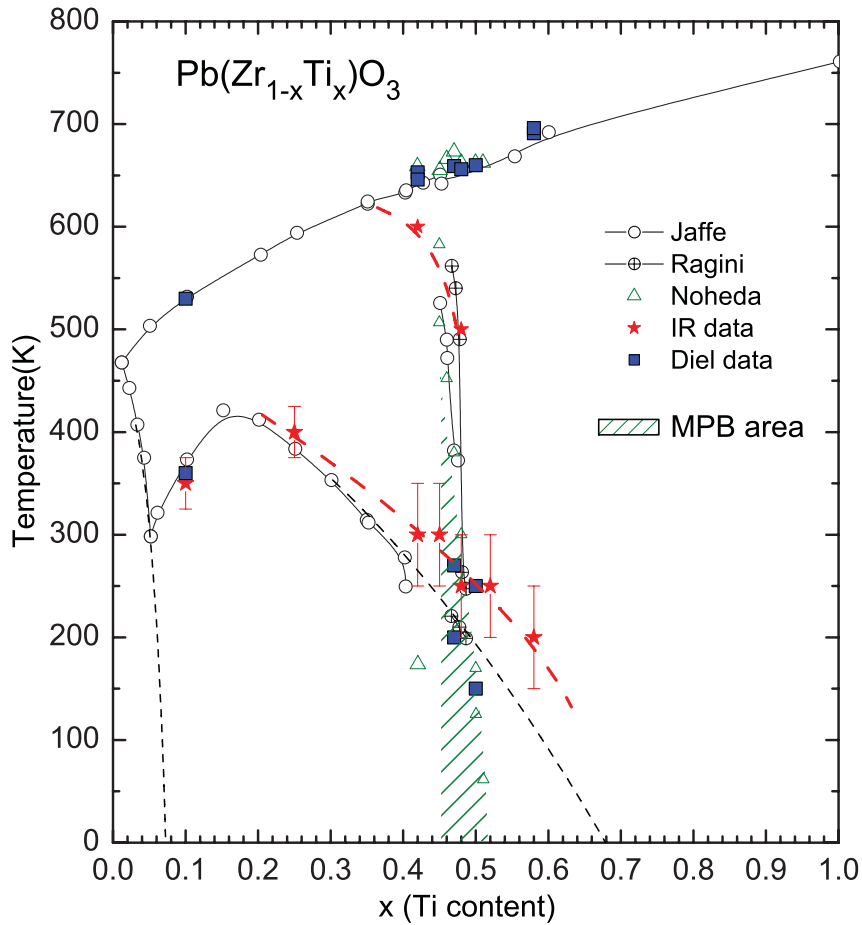

FIG. 9. (Color online) Phase diagram of PZT including our IR and dielectric data compared with data previously published in the literature (Refs. 4, 32, and 37).

Fig. 9. In this diagram, we present our IR data compared with the previous literature and some data from our dielectric measurements. The tilt line found by our IR data appears to be at higher temperatures than the extrapolation found in the literature. The dashed (black) line toward the tetragonal side was extrapolated from dielectric and resonance frequency data measured in poled MPB samples. ${ }^{37}$ The IR technique can resolve the AFD mode when the tilt already occurred in some clusters; therefore, it can be detected at higher temperatures. Our dielectric data revealed some anomalies in the loss spectra at lower temperatures, but these anomalies are not present in the permittivity and they can be due to dielectric relaxations of some extrinsic origin. In MPB samples, the elastic compliance derived from the resonance data changes the sign of the slope at the AFD phase transition temperature. ${ }^{37}$ We performed resonance frequency measurements in PZT 42/58, and the derivative of the elastic compliance changes the slope with temperature near $170 \mathrm{~K}$ but its sign does not; this might indicate a weakening of this transition on the tetragonal side. ${ }^{43}$

The lowest-frequency mode (the $\mathrm{THz}$ mode) seems to have its origin in anharmonic $\mathrm{Pb}$ vibrations (hopping among the available off-center sites). In the cubic phase, its parameters are not too precise due to the limited accuracy of the reflectivity data at high temperatures. In our model, linked to the $\mathrm{THz}$ values at $900 \mathrm{~K}$, the $\mathrm{THz}$ mode is probably a CM component, coupled to a traditional $\mathrm{SM}$ and related to the vibrations of $\mathrm{Pb}$ atoms within a single-wall potential at the displaced (off-centered) sites. In the FE phase, but at higher temperatures (>400 K), we still can assign it to a $\mathrm{CM}$ component $(\mathrm{Pb}$ hopping among the available disordered sites). This behavior has already been found in $\mathrm{PbZrO}_{3}$ ceramics, with a similar 

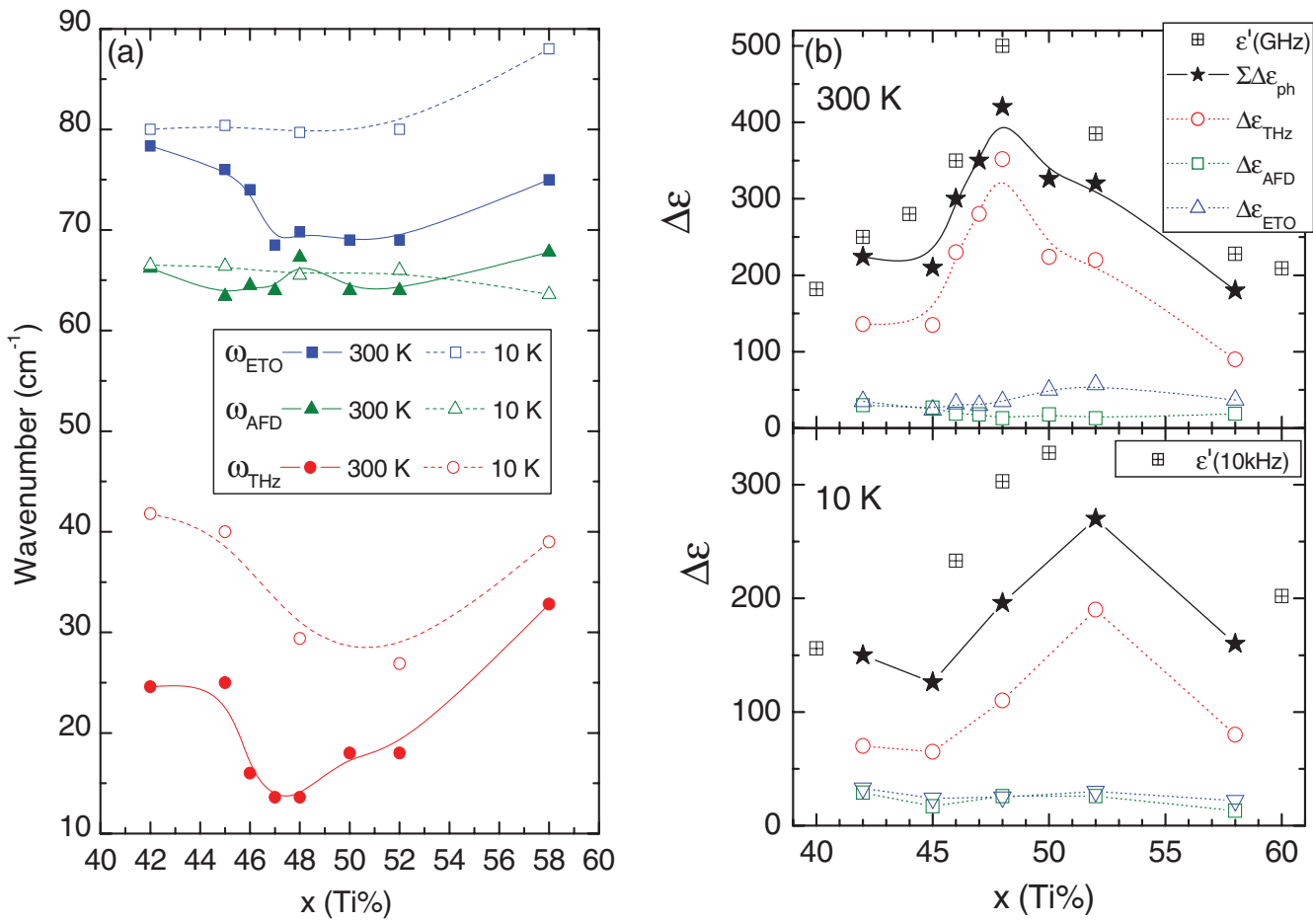

FIG. 10. (Color online) Compositional dependence of the (a) frequency and (b) dielectric contribution of phonons in PZT at $300 \mathrm{~K}$ and 10 K. Crossed symbols correspond to measured permittivity values from Refs. 47 and 48.

frequency of the CM, present above room temperature. ${ }^{44}$ However, it seems there is a change in the nature of the mode with the Ti concentration, because its oscillator strength is lower in tetragonal samples in this temperature range (see Fig. 8). On cooling, the $\mathrm{Pb}$ hopping becomes ever less probable, and below $T_{\text {tilt }}$ we may neglect it. In the phases with doubled cells, a new E-symmetry mode is allowed by symmetry, corresponding to antiphase vibrations of the neighboring $\mathrm{Pb}$ atoms (see Table II).

Figure 10(a) depicts the compositional dependence of the frequencies of the three modes below $100 \mathrm{~cm}^{-1} . \omega_{\mathrm{THz}}$ reveals a minimum for the MPB samples at room temperature, which is shifted toward the higher Ti content sample PZT 48/52 at $10 \mathrm{~K}$. The figure also shows that the AFD mode is present in all compositions already at $300 \mathrm{~K}$.

The room-temperature softening of the lowest-frequency mode toward the MPB in tetragonal PZT samples was already seen by Sivasubramanian et al. ${ }^{24}$ however, they did not measure below $50 \mathrm{~cm}^{-1}$, and the accuracy of their measurements is questionable. Due to this, they did not observe the $\mathrm{THz}$ mode, and they assigned the increase of the permittivity to the ETO mode. From our measurements, it is clear that the main contribution to the permittivity comes from the $\mathrm{THz}$ mode; therefore, the main softening toward MPB takes place in this frequency range.

The composition dependence of the dielectric strengths and of the total phonon contribution $\left(\sum \Delta \varepsilon_{\mathrm{ph}}\right)$ to the permittivity is presented in Fig. 10(b). At room temperature, $\sum \Delta \varepsilon_{\mathrm{ph}}$ shows a clear maximum for the MPB sample. This is caused by the dielectric strength of the $\mathrm{THz}$ mode, $\Delta \varepsilon_{\mathrm{THz}}$, which shows the same maximum, and not by the ETO mode. At low temperatures, the maximum occurs at PZT 48/52; this corroborates that the MPB is shifted to the tetragonal side of the compositional diagram, as suggested in the phase diagram of Ref. 4. Our results demonstrate that the higher values of the intrinsic permittivity in PZT ceramics near the MPB are mainly due to an anharmonic excitation of E-symmetry in the $\mathrm{THz}$ range. This was already calculated by Carl and Hardt ${ }^{45}$ and by Ishibashi and Iwata, ${ }^{46}$ using composition dependence in the coefficients of the Landau-Devonshire free energy. Both showed that there is a divergence of the susceptibility perpendicular to the polar axis at the MPB, i.e., due to the permittivity of E-symmetry vibrations. Nevertheless, this anharmonicity alone cannot explain the high value of the permittivity at room temperature.

Figure 10(b) shows also the permittivity values measured at room temperature in the gigahertz range $\mathrm{e}^{47}$ and at $4.3 \mathrm{~K}$ in the kilohertz range. ${ }^{48}$ At room temperature, the gigahertz values of the permittivity present the same compositional behavior as the extrapolated values from phonons, $\Delta \varepsilon_{\mathrm{ph}}$, except that the gigahertz values are slightly higher. This proves that additional contributions to the permittivity below the $\mathrm{THz}$ range have an extrinsic origin, probably from piezoelectric resonance in grains and vibrations of domain walls; therefore, the $\mathrm{THz}$ range gives the low-frequency limit of the intrinsic permittivity. At even lower frequencies, the extrinsic contributions raise the value of the permittivity to above 1000 at room temperature (see Fig. 7). However, at low temperatures (lower panel in Fig. 10(b)), the value of the permittivity at $10 \mathrm{kHz}$ is just slightly higher than that extrapolated from phonons, and it presents a maximum near $x=0.52$. This means that most of the extrinsic contribution vanishes at low temperatures, and that the MPB is shifted to the tetragonal side of the phase diagram. 


\section{CONCLUSIONS}

The IR and TDTS spectra of several compositions of nominally pure PZT ceramics around the MPB were measured and analyzed. The double occupancy of $\mathrm{Ti}$ and $\mathrm{Zr}$ atoms in the $B$ position of the perovskite lattice and the presence of $\mathrm{Ti} / \mathrm{Zr}$-rich nanoclusters could explain the number of IR active modes through the MPB above room temperature, without the need of macroscopic monoclinic symmetry. A new peak in the IR spectra near $70 \mathrm{~cm}^{-1}$, assigned to an AFD mode, appears in all samples near and below room temperature. Therefore, in the PZT compositional phase diagram, the line corresponding to the octahedra tilted antiferrodistorted phase was extended toward the tetragonal side.

Three main excitations govern the very far IR dielectric spectra of PZT: the lowest mode in the THz range, which corresponds to an anharmonic component (CM) at higher temperatures and gradually transforms into the new activated E-symmetry mode in the tilted phases; the AFD mode near $70 \mathrm{~cm}^{-1}$, activated by its coupling to the polarization on lowering the temperatures; and the E-symmetry SM of the
FE transition. Our measurements confirm that the intrinsic (phonon) permittivity of PZT has a maximum at the MPB, as predicted by theory, although this represents just a small percentage of the total permittivity at lower frequencies, stemming mainly from extrinsic contributions. The permittivity maximum is assigned to the $\mathrm{THz}$ softening of the anharmonic hopping of $\mathrm{Pb}$ atoms among their off-centered positions, perpendicular to the polarization. This maximum is shifted from $x=0.48$ at room temperature to 0.52 at $10 \mathrm{~K}$, meaning that the MPB is shifted toward the tetragonal side of the phase diagram at low temperatures.

\section{ACKNOWLEDGMENTS}

The authors thank B. Noheda and R. Guo for providing some of the PZT samples, M. Savinov and P. Vaněk for dielectric and calorimetric measurements, and J. Hlinka for critical reading of the manuscript and inspiring discussions. This work was supported by the Academy of Sciences of the Czech Republic (Project No. AVOZ10100520) and by the Czech Science Foundation (Project No. P204/10/0616). *buixader@fzu.cz

${ }^{1}$ C. E. Land, P. Thatcher, and G. H. Haertling, in Advances in Materials and Device Research, edited by R. Wolfe, Vol. 4 (Academic Press, New York, 1974), pp. 137.

${ }^{2}$ R. W. Whatmore and A. M. Glazer, J. Phys. C 12, 1505 (1979).

${ }^{3}$ R. Guo, L. E. Cross, S.-E. Park, B. Noheda, D. E. Cox, and G. Shirane, Phys. Rev. Lett. 84, 5423 (2000).

${ }^{4}$ B. Noheda, D. E. Cox, G. Shirane, R. Guo, B. Jones, and L. E. Cross, Phys. Rev. B 63, 014103 (2000).

${ }^{5}$ B. Noheda, J. A. Gonzalo, L. E. Cross, R. Guo, S.-E. Park, D. E. Cox, and G. Shirane, Phys. Rev. B 61, 8687 (2000).

${ }^{6}$ K. A. Schönau, M. Knapp, H. Kungl, M. J. Hoffmann, and H. Fuess, Phys. Rev. B 76, 144112 (2007).

${ }^{7}$ Y. M. Jin, Y. U. Wang, A. G. Khachaturyan, J. F. Li, and D. Viehland, J. Appl. Phys. 94, 3629 (2003).

${ }^{8}$ M. Hinterstein, J. Rouquette, J. Haines, Ph. Papet, M. Knapp, J. Glaum, and H. Fuess, Phys. Rev. Lett. 107, 077602 (2011).

${ }^{9}$ R. Schierholz and H. Fuess, Phys. Rev. B 84, 064122 (2011).

${ }^{10}$ D. Phelan, X. Long, Y. Xie, Z.-G. Ye, A. M. Glazer, H. Yokota, P. A. Thomas, and P. M. Gehring, Phys. Rev. Lett. 105, 207601 (2010).

${ }^{11}$ S. Gorfman, D. S. Keeble, A. M. Glazer, X. Long, Y. Xie, Z.-G. Ye, S. Collins, and P. A. Thomas, Phys. Rev. B 84, 020102 (2011).

${ }^{12}$ A. M. Glazer, P. A. Thomas, K. Z. Baba-Kishi, G. K. H. Pang, and C. W. Tai, Phys. Rev. B 70, 184123 (2004).

${ }^{13}$ A. Bell, J. Mater. Sci. 41, 212 (2006).

${ }^{14}$ G. Burns and B. A. Scott, Phys. Rev. Lett. 25, 1191 (1970).

${ }^{15}$ J. F. Meng, R. S. Katiyar, G. T. Zou, and X. H. Wang, Phys. Status Solidi A 164, 851 (1997).

${ }^{16}$ J. Frantti, J. Lappalainen, V. Lantto, S. Nishio, and M. Kakihana, Jpn. J. Appl. Phys. 38, 5679 (1999).

${ }^{17}$ A. G. Souza-Filho, K. C. V. Lima, A. P. Ayala, I. Guedes, P. T. C. Freire, F. E. A. Melo, J. M. Filho, E. B. Araujo, and J. A. Eiras, Phys. Rev. B 66, 132107 (2002).
${ }^{18}$ J. Rouquette, J. Haines, V. Bornand, M. Pintard, Ph. Papet, and J. L. Sauvajol, J. Phys. Chem. B 73, 224118 (2006).

${ }^{19}$ M. Deluca, H. Fukumura, N. Tonari, C. Capiani Noriyuki Hasuike, C. K. Kisoda, C. Galassi, and H. Harima, J. Raman Spectros. 42, 488 (2010).

${ }^{20}$ E. Buixaderas, I. Gregora, S. Kamba, J. Petzelt, and M. Kosec, J. Phys. Condens. Matter 20, 345229 (2008).

${ }^{21}$ E. Buixaderas, D. Nuzhnyy, P. Vanek, I. Gregora, J. Petzelt, V. Porokhonskyy, L. Jin, and D. Damjanovic, Phase Trans. 83, 917 (2010).

${ }^{22}$ E. Buixaderas, M. Berta, L. Kozielski, and I. Gregora, Phase Trans. 84, 528 (2011).

${ }^{23}$ V. Železný, P. Simon, F. Gervais, and T. Kala, Mat. Res. Bull. 22, 1695 (1987).

${ }^{24}$ V. Sivasubramanian, V. R. K. Murthy, B. Viswanathan, and M. Sieskind, J. Phys. Condens. Matter 8, 2447 (1996).

${ }^{25}$ I. Fedorov, J. Petzelt, V. Zelezny, G. A. Komandin, A. A. Volkov, K. Brooks, Y. Huang, and N. Setter, J. Phys. Condens. Matter 7, 4313 (1995).

${ }^{26}$ E. B. Araújo, K. Yukimitu, J. C. S. Moraes, L. H. Z. Pelaio, and J. A. Eiras, J. Phys. Condens. Matter 14, 5195 (2002).

${ }^{27}$ E. B. Araújo, C. A. Guarany, K. Yukimitu, J. C. S. Moraes, and J. A. Eiras, Ferroelectrics 337, 145 (2006).

${ }^{28}$ C. A. Guarany, L. H. Z. Pelaio, E. B. Araújo, K. Yukimitu, J. C. S. Moraes, and J. A. Eiras, J. Phys. Condens. Matter 15, 4851 (2003).

${ }^{29}$ E. Buixaderas, V. Bovtun, S. Veljko, M. Savinov, P. Kužel, I. Gregora, and S. Kamba, J. Appl. Phys. 108, 104101 (2010).

${ }^{30}$ E. Buixaderas, S. Kamba, J. Petzelt, J. Drahokoupil, F. Laufek, and M. Kosec, Appl. Phys. Lett. 91, 112909 (2007).

${ }^{31}$ M. I. Morozov and D. Damjanovic, J. Appl. Phys. 104, 034107 (2008).

${ }^{32}$ B. Jaffe, W. R. Cook, and H. Jaffe, Piezoelectric Ceramics (Academic Press, New York, 1971), pp. 135. 
${ }^{33} \mathrm{~F}$. Gervais, in Infrared and Millimeter Waves, edited by K. J. Button, Vol. 8 (Academic Press, New York, 1983), pp. 279.

${ }^{34}$ J. Hlinka, J. Petzelt, S. Kamba, D. Noujni, and T. Ostapchuk, Phase Trans. 79, 41 (2006).

${ }^{35}$ M. D. Fontana, H. Idrissi, G. E. Kugel, and K. Wojcik, J. Phys. Condens. Matter 3, 8695 (1991).

${ }^{36}$ E. Buixaderas, S. Kamba, and J. Petzelt, Ferroelectrics 308, 131 (2004).

${ }^{37}$ Ragini, S. K. Mishra, D. Pandey, H. Lemmens, and G. Van Tendeloo, Phys. Rev. B 64, 054101 (2001).

${ }^{38}$ A. J. Bell, J. Mat. Sci. 41, 13 (2006).

${ }^{39}$ A. S. Barker Jr. and A. J. Sievers, Rev. Mod. Phys. 47, S1 (1975).

${ }^{40}$ P. Ghosez, E. Cockayne, U. V. Waghmare, and K. M. Rabe, Phys. Rev. B 60, 836 (1999).
${ }^{41}$ D. Viehland, J.-F. Li, X. Dai, and Z. Xu, J. Phys. Chem. Solids 57, 1545 (1996).

${ }^{42}$ I. A. Kornev, L. Bellaiche, P. E. Janolin, B. Dkhil, and E. Suard, Phys. Rev. Lett. 97, 157601 (2006).

${ }^{43}$ D. Damjanovic (unpublished).

${ }^{44}$ T. Ostapchuk, J. Petzelt, V. Zelezny, S. Kamba, V. Bovtun, V. Porokhonskyy, A. Pashkin, P. Kuzel, M. D. Glinchuk, I. P. Bykov, B. Gorshunov, and M. Dressel, J. Phys. Condens. Matter 13, 2677 (2001).

${ }^{45}$ K. Carl and K. H. Hardtl, Phys. Status Solidi A 8, 87 (1971).

${ }^{46}$ Y. Ishibashi and M. Iwata, Jpn. J. Appl. Phys. 37, L985 (1998).

${ }^{47}$ U. Böttger and G. Arlt, Ferroelectrics 127, 95 (1992).

${ }^{48}$ M. J. Haun, Z. Q. Zhuang, E. Furman, S. J. Jang, and L. E. Cross, Ferroelectrics 99, 45 (1989). 